\title{
Determining The Magnetic Potential In The Fractional Magnetic Calderón Problem
}

\author{
$\mathrm{Li} \mathrm{Li}$ \\ Department of Mathematics, University of Washington, \\ Seattle, WA 98195, USA
}

\begin{abstract}
We determine both the magnetic potential and the electric potential from the exterior partial measurements of the Dirichlet-to-Neumann map in the fractional linear magnetic Calderón problem by using an integral identity. We also determine both the magnetic potential and the nonlinearity in the fractional semilinear magnetic Calderón problem by using a first order linearization.
\end{abstract}

\section{Introduction}

In this paper we continue the study of the fractional magnetic Calderón problem introduced in [14, which is a generalization of the fractional Calderón problem introduced in [6, 8] as well as a nonlocal analogue of the classical magnetic Calderón problem studied in [4, 10, 16, 19].

We consider the operator $\mathcal{R}_{A}^{s}$, which is formally defined by

$$
\mathcal{R}_{A}^{s} u(x):=2 \lim _{\epsilon \rightarrow 0^{+}} \int_{\mathbb{R}^{n} \backslash B_{\epsilon}(x)}\left(u(x)-R_{A}(x, y) u(y)\right) K(x, y) d y
$$

where $K$ is a function associated with a heat kernel (see Subsection 2.2 in [14) satisfying

$$
K(x, y)=K(y, x), \quad K(x, y) \sim \frac{1}{|x-y|^{n+2 s}},
$$

$A$ is a real vector-valued magnetic potential and

$$
R_{A}(x, y):=\cos \left((x-y) \cdot A\left(\frac{x+y}{2}\right)\right)
$$

For real-valued $u, \mathcal{R}_{A}^{s}$ is the real part of the fractional operator $\mathcal{L}_{A}^{s}$ introduced in [14] and clearly we have $\mathcal{R}_{A}^{s}=\mathcal{R}_{-A}^{s}$.

Under appropriate assumptions on $A$ and the electric potential $q$, the exterior Dirichlet problem

$$
\left\{\begin{aligned}
\left(\mathcal{R}_{A}^{s}+q\right) u=0 & \text { in } \Omega \\
u=g & \text { in } \Omega_{e}
\end{aligned}\right.
$$


is well-posed so we can define the solution operator $P_{A, q}: g \rightarrow u_{g}$ and the Dirichlet-to-Neumann map $\Lambda_{A, q}$, which is formally given by

$$
\Lambda_{A, q} g:=\left.\mathcal{R}_{A}^{s}\left(P_{A, q} g\right)\right|_{\Omega_{e}} .
$$

In [14, we determined $q$ from the exterior partial measurements of $\Lambda_{A, q}$ for fixed $A$. Our goal here is to determine both $A$ and $q$ from the knowledge of the Dirichlet-to-Neumann map. The following theorem is our first main result in this paper.

Theorem 1.1. Suppose $\operatorname{supp} A_{j} \subset \Omega \subset B_{r}(0)$ for some constant $r>0, c \leq q_{j} \in L^{\infty}(\Omega)$ for some constant $c>0, A_{j} \in L^{\infty}\left(\mathbb{R}^{n}\right), W_{j}$ are open sets s.t. $W_{j} \cap B_{3 r}(0)=\emptyset(j=1,2)$. Let

$$
W^{(1,2)}=\left\{\frac{x+y}{2}: x \in W_{1}, y \in W_{2}\right\} .
$$

Also assume $W^{(1,2)} \backslash\left(\operatorname{supp} A_{1} \cup \operatorname{supp} A_{2}\right) \neq \emptyset$. If

$$
\left.\Lambda_{A_{1}, q_{1}} g\right|_{W_{2}}=\left.\Lambda_{A_{2}, q_{2}} g\right|_{W_{2}}
$$

for any $g \in C_{c}^{\infty}\left(W_{1}\right)$, then $A_{1}= \pm A_{2}$ and $q_{1}=q_{2}$.

Remark. Recall that in the classical magnetic Calderón problem, it is impossible to completely determine A from the knowledge of the Dirichlet-to-Neumann map since the Dirichlet-to-Neumann maps associated with $\left(A_{1}, q\right)$ and $\left(A_{2}, q\right)$ coincide whenever $A_{1}$ and $A_{2}$ are gauge equivalent, i.e.

$$
A_{1}-A_{2}=\nabla \phi
$$

for some smooth $\phi$ in $\bar{\Omega}$ with $\left.\phi\right|_{\partial \Omega}=0$. However in this fractional magnetic Calderón problem, we are able to completely determine A (up to the sign) from the knowledge of $\Lambda_{A, q}$. See [1] for the study of a different fractional magnetic Calderón problem and see [2] for a recent study connecting local and nonlocal magnetic Calderón problems. More results for the fractional linear Calderón problem can be found in [7, 17].

In this paper we also continue the study of the fractional semilinear magnetic Calderón problem introduced in [15, which is a generalization of the fractional semilinear Calderón problem introduced in [12] as well as a semilinear analogue of the inverse problem studied in [14. See [9, 11, 13, for results for the classical semilinear Calderón problem.

As in [15], we focus on $K(x, y)=c_{n, s} /|x-y|^{n+2 s}$ in the fractional semilinear Calderón problem. In this case, $\mathcal{R}_{A}^{s}$ is the real part of the fractional magnetic Laplacian $(-\Delta)_{A}^{s}$ studied in [3, 18, for real-valued $u$.

We consider the nonlinear exterior Dirichlet problem

$$
\left\{\begin{array}{rlrl}
\mathcal{R}_{A}^{s} u+a(x, u) & =0 & & \text { in } \Omega \\
u=g & & \text { in } \Omega_{e}
\end{array}\right.
$$

where the nonlinearity $a(x, z): \Omega \times \mathbb{R} \rightarrow \mathbb{R}$ satisfies

(i) $z \rightarrow a(\cdot, z)$ is analytic with values in the Hölder space $C^{s}(\Omega)$;

(ii) $a(x, 0)=0$ and $\partial_{z} a(x, 0) \geq c>0$ for some constant $c>0$

so we have the Taylor's expansion

$$
a(x, z)=\sum_{k=1}^{\infty} a_{k}(x) \frac{z^{k}}{k !}, \quad a_{k}(x)=\partial_{z}^{k} a(x, 0) \in C^{s}(\Omega)
$$


where the series converges in $C^{s}(\Omega)$ topology.

Under some boundedness conditions on $A$, (6) is well-posed for small $g$ so we can define the solution operator $Q_{A, a}: g \rightarrow u_{g}$ and the Dirichlet-to-Neumann map $\Lambda_{A, a}$, which is formally given by

$$
\Lambda_{A, a} g:=\left.\mathcal{R}_{A}^{s}\left(Q_{A, a} g\right)\right|_{\Omega_{e}} .
$$

In [15, we determined $a$ from the exterior partial measurements of $\Lambda_{A, a}$ for fixed $A$. Our next goal here is to determine both $A$ and $a$ from the knowledge of the Dirichlet-to-Neumann map. The following theorem is our second main result in this paper.

Theorem 1.2. Suppose $\operatorname{supp} A_{j} \subset \Omega \subset B_{r}(0)$ for some constant $r>0$ and $\left\|A_{j}\right\|_{L^{\infty}\left(\mathbb{R}^{n}\right)} \leq$ $\pi /(8 \sqrt{n} r), a^{(j)}$ satisfy (i) and (ii), $W_{j}$ are open sets s.t. $W_{j} \cap B_{3 r}(0)=\emptyset(j=1,2)$. Let

$$
W^{(1,2)}=\left\{\frac{x+y}{2}: x \in W_{1}, y \in W_{2}\right\} .
$$

Also assume $W^{(1,2)} \backslash\left(\operatorname{supp} A_{1} \cup \operatorname{supp} A_{2}\right) \neq \emptyset$. If

$$
\left.\Lambda_{A_{1}, a^{(1)}} g\right|_{W_{2}}=\left.\Lambda_{A_{2}, a^{(2)}} g\right|_{W_{2}}, \quad g \in C_{c}^{\infty}\left(W_{1}\right)
$$

whenever $\|g\|_{C^{2}\left(\mathbb{R}^{n}\right)}$ is sufficiently small, then $A_{1}= \pm A_{2}$ in $\Omega$ and $a^{(1)}=a^{(2)}$ in $\Omega \times \mathbb{R}$.

The rest of this paper is organized in the following way. In Section 2, we summarize the background knowledge. We prove Theorem 1.1 by using an integral identity in Section 3. Based on Theorem 1.1, we prove Theorem 1.2 by using a first order linearization in the Sobolev space $H^{s}\left(\mathbb{R}^{n}\right)$ in Section 4.

Acknowledgement. The author is partly supported by National Science Foundation. The author would like to thank Professor Gunther Uhlmann for suggesting the problem and for helpful discussions.

\section{Preliminaries}

Throughout this paper

- $n \geq 2$ denotes the space dimension and $0<s<1$ denotes the fractional power

- $\Omega$ denotes a bounded domain with $C^{1,1}$ boundary and $\Omega_{e}:=\mathbb{R}^{n} \backslash \bar{\Omega}$

- $B_{r}(0)$ denotes the open ball centered at the origin with radius $r>0$

- $A: \mathbb{R}^{n} \rightarrow \mathbb{R}^{n}$ denotes a real vector-valued magnetic potential

- $c, C, C^{\prime}, C_{1}, \cdots$ denote positive constants (which may depend on some parameters but always independent of small constants $\epsilon, \rho$ )

- $\int \cdots \int=\int_{\mathbb{R}^{n}} \cdots \int_{\mathbb{R}^{n}}$

- $X^{*}$ denotes the continuous dual space of $X$ and write $\langle f, u\rangle=f(u)$ for $u \in X, f \in X^{*}$

- $\|\cdot\|_{C^{2}\left(\mathbb{R}^{n}\right)}$ is defined by

$$
\|f\|_{C^{2}\left(\mathbb{R}^{n}\right)}=\sum_{|\alpha| \leq 2}\left\|\partial^{\alpha} f\right\|_{L^{\infty}\left(\mathbb{R}^{n}\right)}
$$




\section{$2.1 \quad$ Function Spaces}

Throughout this paper we refer all function spaces to real-valued function spaces.

For $t \in \mathbb{R}, H^{t}\left(\mathbb{R}^{n}\right)$ denotes the Sobolev space $W^{t, 2}\left(\mathbb{R}^{n}\right)$.

We have the natural identification

$$
H^{-t}\left(\mathbb{R}^{n}\right)=H^{t}\left(\mathbb{R}^{n}\right)^{*}
$$

Let $U$ be an open set and $F$ be a closed set in $\mathbb{R}^{n}$,

$$
\begin{gathered}
H^{t}(U):=\left\{\left.u\right|_{U}: u \in H^{t}\left(\mathbb{R}^{n}\right)\right\}, \quad H_{F}^{t}\left(\mathbb{R}^{n}\right):=\left\{u \in H^{t}\left(\mathbb{R}^{n}\right): \operatorname{supp} u \subset F\right\}, \\
\tilde{H}^{t}(U):=\text { the closure of } C_{c}^{\infty}(U) \text { in } H^{t}\left(\mathbb{R}^{n}\right) .
\end{gathered}
$$

Since $\Omega$ is a bounded domain with $C^{1,1}$ boundary implies $\Omega$ is Lipschitz bounded,

$$
\tilde{H}^{t}(\Omega)=H_{\bar{\Omega}}^{t}\left(\mathbb{R}^{n}\right) .
$$

For $0<s<1, C^{s}(U)$ denotes the Hölder space $C^{0, s}(U)$.

\subsection{Old Results}

All the results presented in this subsection can be found in [14] and [15].

We have the bilinear form definition of $\mathcal{R}_{A}^{s}$, which is given by

$$
\left\langle\mathcal{R}_{A}^{s} u, v\right\rangle=2 \iint\left(u(x)-R_{A}(x, y) u(y)\right) v(x) K(x, y) d x d y .
$$

Proposition 2.1. Suppose $0<s<1$ and $A \in L^{\infty}\left(\mathbb{R}^{n}\right)$, then the operator $\mathcal{R}_{A}^{s}: H^{s}\left(\mathbb{R}^{n}\right) \rightarrow$ $H^{-s}\left(\mathbb{R}^{n}\right)$ is linear, bounded and

$$
\left\langle\mathcal{R}_{A}^{s} u, v\right\rangle=\left\langle\mathcal{R}_{A}^{s} v, u\right\rangle .
$$

We have the well-posedness of the linear and semilinear exterior Dirichlet problems.

Proposition 2.2. Suppose $A \in L^{\infty}\left(\mathbb{R}^{n}\right)$ and $c \leq q \in L^{\infty}(\Omega)$, then the linear exterior problem (3) has a unique (weak) solution $u_{g} \in H^{s}\left(\mathbb{R}^{n}\right)$ for each $g \in H^{s}\left(\mathbb{R}^{n}\right)$ and the solution operator $P_{A, q}$ is bounded on $H^{s}\left(\mathbb{R}^{n}\right)$.

Proposition 2.3. Suppose $\operatorname{supp} A \subset \Omega \subset B_{r}(0)$ for some $r>0$ and $\|A\|_{L^{\infty}\left(\mathbb{R}^{n}\right)} \leq \pi /(8 \sqrt{n} r)$, $W \cap B_{3 r}(0)=\emptyset$ and $g \in C_{c}^{\infty}(W)$. There exists a small constant $\rho>0$ s.t. if $\|g\|_{C^{2}\left(\mathbb{R}^{n}\right)} \leq \rho$, then the semilinear exterior problem (6) has a unique solution $u \in H^{s}\left(\mathbb{R}^{n}\right) \cap C^{s}\left(\mathbb{R}^{n}\right)$ satisfying

$$
\left(u-P_{A, a_{1}} g\right) \in M:=\left\{v \in C^{s}\left(\mathbb{R}^{n}\right):\left.v\right|_{\Omega_{e}}=0,\|v\|_{C^{s}\left(\mathbb{R}^{n}\right)} \leq \rho\right\} .
$$

Denote the associated solution operator by $Q_{A, a}$. Moreover, we have

$$
\left\|Q_{A, a} g\right\|_{C^{s}\left(\mathbb{R}^{n}\right)} \leq C\|g\|_{C^{2}\left(\mathbb{R}^{n}\right)} .
$$


The Dirichlet-to-Neumann map $\Lambda_{A, q}$ associated with (3) can be defined by a bilinear form on $H^{s}\left(\Omega_{e}\right) \times H^{s}\left(\Omega_{e}\right)$. For $g, h \in C_{c}^{\infty}\left(\Omega_{e}\right)$, we have

$$
\left\langle\Lambda_{A, q} g, h\right\rangle=\left\langle\mathcal{R}_{A}^{s}\left(P_{A, q} g\right), h^{*}\right\rangle+\int_{\Omega}\left(P_{A, q} g\right) h^{*}
$$

where $h^{*} \in H^{s}\left(\mathbb{R}^{n}\right)$ satisfying $h^{*}-h \in \tilde{H}^{s}(\Omega)$. We know that (12) does not depend on the choice of $h^{*}$ and

$$
\left\langle\Lambda_{A, q} g, h\right\rangle=\left\langle\Lambda_{A, q} h, g\right\rangle .
$$

This bilinear form definition coincides with the definition given by (4).

The Dirichlet-to-Neumann map $\Lambda_{A, a}$ associated with (6) cannot be defined by a bilinear form due to the nonlinearity $a(\cdot, \cdot)$. Proposition 2.3 ensures that (8) is well-defined at least for $g$ satisfying the condition assumed in the statement of the proposition.

We also have the following Runge approximation property.

Proposition 2.4. Suppose $\operatorname{supp} A \subset \Omega \subset B_{r}(0)$ for some $r>0, W$ is an open set s.t. $W \subset \Omega_{e}$ and $W \cap B_{3 r}(0)=\emptyset$, then

$$
S:=\left\{\left.P_{A, q} f\right|_{\Omega}: f \in C_{c}^{\infty}(W)\right\}
$$

is dense in $L^{2}(\Omega)$.

We will prove Theorem 1.1 based on the following theorem in [14.

Theorem 2.5. Suppose $\operatorname{supp} A \subset \Omega \subset B_{r}(0)$ for some constant $r>0, c \leq q_{j} \in L^{\infty}(\Omega)$ for some constant $c>0, A \in L^{\infty}\left(\mathbb{R}^{n}\right), W_{j}$ are open sets s.t. $W_{j} \cap B_{3 r}(0)=\emptyset(j=1,2)$. If

$$
\left.\Lambda_{A, q_{1}} g\right|_{W_{2}}=\left.\Lambda_{A, q_{2}} g\right|_{W_{2}}
$$

for any $g \in C_{c}^{\infty}\left(W_{1}\right)$, then $q_{1}=q_{2}$.

The following first order linearization relates the semilinear problem to the linear one.

Proposition 2.6. Suppose $\operatorname{supp} A \subset \Omega \subset B_{r}(0)$ for some $r>0$ and $\|A\|_{L^{\infty}\left(\mathbb{R}^{n}\right)} \leq \pi /(8 \sqrt{n} r)$, $W \cap B_{3 r}(0)=\emptyset$ and $g \in C_{c}^{\infty}(W)$, then

$$
Q_{A, a}(\epsilon g) / \epsilon \rightarrow P_{A, a_{1}} g
$$

in $H^{s}\left(\mathbb{R}^{n}\right)$ as $\epsilon \rightarrow 0$.

We will use the first order linearization above to prove Theorem 1.2 based on the following theorem in [15].

Theorem 2.7. Suppose $\operatorname{supp} A \subset \Omega \subset B_{r}(0)$ for some constant $r>0$ and $\|A\|_{L^{\infty}(\Omega)} \leq \pi /(8 \sqrt{n} r)$, $a^{(j)}$ satisfy (i) and (ii), $W_{j}$ are open sets s.t. $W_{j} \cap B_{3 r}(0)=\emptyset(j=1,2)$. If

$$
\left.\Lambda_{A, a^{(1)}} g\right|_{W_{2}}=\left.\Lambda_{A, a^{(2)}} g\right|_{W_{2}}, \quad g \in C_{c}^{\infty}\left(W_{1}\right)
$$

whenever $\|g\|_{C^{2}\left(\mathbb{R}^{n}\right)}$ is sufficiently small, then $a^{(1)}=a^{(2)}$ in $\Omega \times \mathbb{R}$.

Remark. Compared with the statement of Theorem 2.5 (respectively, Theorem 2.7), the statement of Theorem 1.1 (respectively, Theorem 1.2) contains an additional assumption on the set $W^{(1,2)}$. This assumption ensures that the double integral in the integral identity (obtained in Section 3) is actually over the region $\Omega \times \Omega$, which enables us to apply the Runge approximation property (Proposition 2.4). See the proof in Section 3 for details. 


\section{The Proof of Theorem 1.1}

We first build an integral identity, which will be useful in the proof of Theorem 1.1.

For $g_{j} \in C_{c}^{\infty}\left(\Omega_{e}\right)$ and $u_{j}=P_{A_{j}, q_{j}} g_{j}(j=1,2)$ solving

$$
\left\{\begin{aligned}
\left(\mathcal{R}_{A_{j}}^{s}+q_{j}\right) u=0 & \text { in } \Omega \\
u & =g_{j} \quad \text { in } \Omega_{e},
\end{aligned}\right.
$$

by (10), (11), (12) and (13) we have

$$
\begin{gathered}
\left\langle\left(\Lambda_{A_{1}, q_{1}}-\Lambda_{A_{2}, q_{2}}\right) g_{1}, g_{2}\right\rangle=\left\langle\Lambda_{A_{1}, q_{1}} g_{1}, g_{2}\right\rangle-\left\langle\Lambda_{A_{2}, q_{2}} g_{2}, g_{1}\right\rangle \\
=\left\langle\mathcal{R}_{A_{1}}^{s} u_{1}, u_{2}\right\rangle+\int_{\Omega} q_{1} u_{1} u_{2}-\left\langle\mathcal{R}_{A_{2}}^{s} u_{2}, u_{1}\right\rangle-\int_{\Omega} q_{2} u_{2} u_{1} \\
=\left\langle\mathcal{R}_{A_{1}}^{s} u_{1}, u_{2}\right\rangle-\left\langle\mathcal{R}_{A_{2}}^{s} u_{1}, u_{2}\right\rangle-\int_{\Omega}\left(q_{2}-q_{1}\right) u_{1} u_{2} \\
=\iint 2\left(R_{A_{2}}(x, y)-R_{A_{1}}(x, y)\right) K(x, y) u_{1}(y) u_{2}(x) d x d y-\int_{\Omega}\left(q_{2}-q_{1}\right) u_{1} u_{2} .
\end{gathered}
$$

Now we are ready to prove Theorem 1.1.

Proof. For $g_{j} \in C_{c}^{\infty}\left(W_{j}\right)$ and $u_{j}=P_{A_{j}, q_{j}} g_{j}(j=1,2)$, by (5) and (14) we have

$$
\iint G(x, y) u_{1}(y) u_{2}(x) d x d y=\int_{\Omega}\left(q_{2}-q_{1}\right) u_{1} u_{2}
$$

where we write

$$
G(x, y):=2\left(R_{A_{2}}(x, y)-R_{A_{1}}(x, y)\right) K(x, y) .
$$

Note that supp $u_{j} \subset \Omega \cup W_{j}$ so the double integral on the left hand side is

$$
\int_{\Omega \cup W_{2}} \int_{\Omega \cup W_{1}} G(x, y) u_{1}(y) u_{2}(x) d x d y=I_{1}+I_{2}+I_{3}+I_{4}
$$

where we write

$$
I_{1}:=\int_{\Omega} \int_{\Omega}, \quad I_{2}:=\int_{\Omega} \int_{W_{1}}, \quad I_{3}:=\int_{W_{2}} \int_{\Omega}, \quad I_{4}:=\int_{W_{2}} \int_{W_{1}} .
$$

Note that $(x, y) \in W_{2} \times \Omega$ (or $\left.(x, y) \in \Omega \times W_{1}\right)$ implies $(x+y) / 2 \geq r, R_{A_{1}}(x, y)=R_{A_{2}}(x, y)=1$, $G(x, y)=0$ so $I_{2}=I_{3}=0$.

Also note that by the assumption on $W^{(1,2)}$, we can choose $x_{0} \in W_{2}, y_{0} \in W_{1}$ s.t. $\frac{x_{0}+y_{0}}{2} \notin$ $\operatorname{supp} A_{j}$ so $(x, y) \in W_{2} \times W_{1}$ implies $(x+y) / 2 \notin \operatorname{supp} A_{j}$ if we replace $W_{2}, W_{1}$ by a small open ball centered at $x_{0}$ and a small open ball centered at $y_{0}$ when necessary. Hence $I_{4}=0$ so we have

$$
\int_{\Omega} \int_{\Omega} G(x, y) u_{1}(y) u_{2}(x) d x d y=\int_{\Omega}\left(q_{2}-q_{1}\right) u_{1} u_{2} .
$$

Now fix open sets $\Omega_{j} \subset \Omega$ s.t. $\Omega_{1} \cap \Omega_{2}=\emptyset$. Also fix $\phi_{j} \in C_{c}^{\infty}\left(\Omega_{j}\right)$ and $\epsilon>0$. 
By Proposition 2.4, we can choose $g_{1} \in C_{c}^{\infty}\left(W_{1}\right)$ s.t.

$$
\left\|u_{1}-\phi_{1}\right\|_{L^{2}(\Omega)} \leq \epsilon
$$

and for this chosen $g_{1}$, we can choose $g_{2} \in C_{c}^{\infty}\left(W_{2}\right)$ s.t.

$$
\left\|u_{1}\right\|_{L^{2}(\Omega)}\left\|u_{2}-\phi_{2}\right\|_{L^{2}(\Omega)} \leq \epsilon
$$

Note that $\phi_{1}(x) \phi_{2}(x)=0$ for $x \in \Omega$ so

$$
\begin{gathered}
\left|\int_{\Omega}\left(q_{2}-q_{1}\right) u_{1} u_{2}\right|=\left|\int_{\Omega}\left(q_{2}-q_{1}\right)\left(u_{1}-\phi_{1}\right) \phi_{2}+\int_{\Omega}\left(q_{2}-q_{1}\right) u_{1}\left(u_{2}-\phi_{2}\right)\right| \\
\leq\left\|\left(q_{2}-q_{1}\right)||_{L^{\infty}}\right\| \phi_{2}\left\|_{L^{2}}\right\| u_{1}-\phi_{1}\left\|_{L^{2}}+\right\|\left(q_{2}-q_{1}\right)\left\|_{L^{\infty}}\right\| u_{1}||_{L^{2}}\left\|u_{2}-\phi_{2}\right\|_{L^{2}} \leq C \epsilon .
\end{gathered}
$$

Also note that

$$
\begin{gathered}
|G(x, y)| \leq 4\left|\sin \left(\frac{x-y}{2} \cdot\left(A_{1}-A_{2}\right)\left(\frac{x+y}{2}\right)\right) \sin \left(\frac{x-y}{2} \cdot\left(A_{1}+A_{2}\right)\left(\frac{x+y}{2}\right)\right)\right| K(x, y) \\
\leq C_{A}|x-y|^{2} K(x, y) \leq \frac{C}{|x-y|^{n+2 s-2}}
\end{gathered}
$$

which implies

$$
\int_{\Omega}|G(x, y)| d y \leq C_{0}, x \in \Omega, \quad \int_{\Omega}|G(x, y)| d x \leq C_{0}, y \in \Omega .
$$

By the generalized Young's Inequality (see Proposition 0.10 on page 9 in [5]),

$$
\|T f\|_{L^{2}(\Omega)} \leq C_{0}\|f\|_{L^{2}(\Omega)}, \quad(T f)(x):=\int_{\Omega}|G(x, y) f(y)| d y
$$

so we have

$$
\begin{gathered}
\left|\int_{\Omega} \int_{\Omega} G(x, y) u_{1}(y) u_{2}(x) d x d y-\int_{\Omega_{1}} \int_{\Omega_{2}} G(x, y) \phi_{1}(y) \phi_{2}(x) d x d y\right| \\
=\left|\int_{\Omega} \int_{\Omega} G(x, y)\left(u_{1}(y)-\phi_{1}(y)\right) \phi_{2}(x) d x d y+\int_{\Omega} \int_{\Omega} G(x, y) u_{1}(y)\left(u_{2}(x)-\phi_{2}(x)\right) d x d y\right| \\
\leq \int_{\Omega} \int_{\Omega}\left|G(x, y) \phi_{2}(x)\right| d x\left|u_{1}(y)-\phi_{1}(y)\right| d y+\int_{\Omega} \int_{\Omega}\left|G(x, y) u_{1}(y)\right| d y\left|u_{2}(x)-\phi_{2}(x)\right| d x \\
\leq C_{0}\left\|\phi_{2}\right\|_{L^{2}}\left\|u_{1}-\phi_{1}\right\|_{L^{2}}+C_{0}\left\|u_{1}\right\|_{L^{2}}\left\|u_{2}-\phi_{2}\right\|_{L^{2}} \leq C^{\prime} \epsilon .
\end{gathered}
$$

Combine (16), (17) with (15). Since $\epsilon$ is arbitrary,

$$
\int_{\Omega_{1}} \int_{\Omega_{2}} G(x, y) \phi_{1}(y) \phi_{2}(x) d x d y=0 .
$$

Note that the set

$$
\left\{\phi_{1} \otimes \phi_{2}: \phi_{j} \in C_{c}^{\infty}\left(\Omega_{j}\right), j=1,2\right\}
$$

generates a space dense in $C_{c}^{\infty}\left(\Omega_{1} \times \Omega_{2}\right)$ so $G(x, y)=0$ in $\Omega_{1} \times \Omega_{2}$. Since $\Omega_{1}, \Omega_{2}$ are arbitrary, $G(x, y)=0$ for $x, y \in \Omega$ whenever $x \neq y$ so

$$
R_{A_{1}}(x, y)=R_{A_{2}}(x, y), \quad x, y \in \Omega .
$$


Now fix $x_{0} \in \Omega$. Let $A^{(k)}$ denote the $k^{t h}$ component of $A$ and let $\left\{e_{k}\right\}_{k=1}^{n}$ denote the standard basis of the vector space $\mathbb{R}^{n}$. Consider $x=x_{0}+\epsilon e_{k}$ and $y=x_{0}-\epsilon e_{k}$ for small $\epsilon>0$. Since $\left|2 \epsilon A_{j}^{(k)}\left(x_{0}\right)\right|<\frac{\pi}{2}, R_{A_{1}}(x, y)=R_{A_{2}}(x, y)$ implies $\left|A_{1}^{(k)}\left(x_{0}\right)\right|=\left|A_{2}^{(k)}\left(x_{0}\right)\right|$.

Suppose there exist $l \neq k$ s.t. $A_{1}^{(k)}\left(x_{0}\right)=A_{2}^{(k)}\left(x_{0}\right) \neq 0$ and $A_{1}^{(l)}\left(x_{0}\right)=-A_{2}^{(l)}\left(x_{0}\right) \neq 0$. Consider $x=x_{0}+\epsilon\left(e_{k}+e_{l}\right)$ and $y=x_{0}-\epsilon\left(e_{k}+e_{l}\right)$, then

$$
(x-y) \cdot A_{j}\left(\frac{x+y}{2}\right)=2 \epsilon\left(A_{j}^{(k)}\left(x_{0}\right)+A_{j}^{(l)}\left(x_{0}\right)\right),
$$

which contradicts with $R_{A_{1}}(x, y)=R_{A_{2}}(x, y)$. Hence the only possibility is $A_{1}\left(x_{0}\right)= \pm A_{2}\left(x_{0}\right)$. Now we have shown $A_{1}= \pm A_{2}$ then Theorem 1.1 is an immediate consequence of Theorem 2.5.

\section{The Proof of Theorem 1.2}

Now we use the first order linearization in $H^{s}\left(\mathbb{R}^{n}\right)$ to prove Theorem 1.2 based on Theorem 1.1.

Proof. For $g \in C_{c}^{\infty}\left(W_{1}\right)$ and small $\epsilon>0, u_{\epsilon, g}^{(j)}=Q_{A_{j}, a^{(j)}}(\epsilon g)$ solve

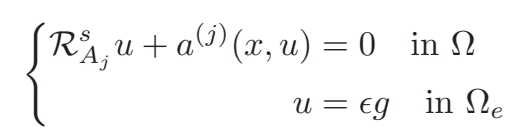

and $u_{g}^{(j)}=P_{A_{j}, a_{1}^{(j)}} g$ solve

$$
\left\{\begin{aligned}
\mathcal{R}_{A_{j}}^{s} u+a_{1}^{(j)}(x) u & =0 & & \text { in } \Omega \\
u & =g & & \text { in } \Omega_{e}
\end{aligned}\right.
$$

$(j=1,2)$. By Proposition 2.6, we have

$$
u_{\epsilon, g}^{(j)} / \epsilon \rightarrow u_{g}^{(j)} \text { in } H^{s}\left(\mathbb{R}^{n}\right),
$$

which implies

$$
\left.\left.\frac{1}{\epsilon} \mathcal{R}_{A_{j}}^{s} u_{\epsilon, g}^{(j)}\right|_{W_{2}} \rightarrow \mathcal{R}_{A_{j}}^{s} u_{g}^{(j)}\right|_{W_{2}} \quad \text { in } H^{-s}\left(W_{2}\right) .
$$

Note that (9) implies

$$
\left.\mathcal{R}_{A_{1}}^{s} u_{\epsilon, g}^{(1)}\right|_{W_{2}}=\left.\mathcal{R}_{A_{2}}^{s} u_{\epsilon, g}^{(2)}\right|_{W_{2}} .
$$

Let $\epsilon \rightarrow 0$, then we have

$$
\left.\mathcal{R}_{A_{1}}^{s} u_{g}^{(1)}\right|_{W_{2}}=\left.\mathcal{R}_{A_{2}}^{s} u_{g}^{(2)}\right|_{W_{2}},
$$

i.e.

$$
\left.\Lambda_{A_{1}, a_{1}^{(1)}} g\right|_{W_{2}}=\left.\Lambda_{A_{2}, a_{1}^{(2)}} g\right|_{W_{2}}, \quad g \in C_{c}^{\infty}\left(W_{1}\right) .
$$

By Theorem 1.1, $A_{1}= \pm A_{2}$. Now Theorem 1.2 is an immediate consequence of Theorem 2.7. 


\section{References}

[1] Giovanni Covi. An inverse problem for the fractional Schrödinger equation in a magnetic field. Inverse Problems, 36(4):045004, 2020.

[2] Giovanni Covi and Angkana Rüland. On some partial data Calderón type problems with mixed boundary conditions. arXiv preprint arXiv:2006.03252, 2020.

[3] Pietro d'Avenia and Marco Squassina. Ground states for fractional magnetic operators. ESAIM: Control, Optimisation and Calculus of Variations, 24(1):1-24, 2018.

[4] David Dos Santos Ferreira, Carlos E Kenig, Johannes Sjöstrand, and Gunther Uhlmann. Determining a magnetic Schrödinger operator from partial cauchy data. Communications in mathematical physics, 271(2):467-488, 2007.

[5] Gerald B Folland. Introduction to partial differential equations. Princeton university press, 1995.

[6] Tuhin Ghosh, Yi-Hsuan Lin, and Jingni Xiao. The Calderón problem for variable coefficients nonlocal elliptic operators. Communications in Partial Differential Equations, 42(12):19231961, 2017.

[7] Tuhin Ghosh, Angkana Rüland, Mikko Salo, and Gunther Uhlmann. Uniqueness and reconstruction for the fractional calderón problem with a single measurement. Journal of Functional Analysis, page 108505, 2020.

[8] Tuhin Ghosh, Mikko Salo, and Gunther Uhlmann. The Calderón problem for the fractional Schrödinger equation. Analysis \& PDE, 13(2):455-475, 2020.

[9] Victor Isakov and John Sylvester. Global uniqueness for a semilinear elliptic inverse problem. Communications on Pure and Applied Mathematics, 47(10):1403-1410, 1994.

[10] Katsiaryna Krupchyk and Gunther Uhlmann. Uniqueness in an inverse boundary problem for a magnetic Schrödinger operator with a bounded magnetic potential. Communications in Mathematical Physics, 327(3):993-1009, 2014.

[11] Katya Krupchyk and Gunther Uhlmann. A remark on partial data inverse problems for semilinear elliptic equations. Proceedings of the American Mathematical Society, 148(2):681-685, 2020.

[12] Ru-Yu Lai and Yi-Hsuan Lin. Inverse problems for fractional semilinear elliptic equations. arXiv preprint arXiv:2004.00549, 2020.

[13] Matti Lassas, Tony Liimatainen, Yi-Hsuan Lin, and Mikko Salo. Inverse problems for elliptic equations with power type nonlinearities. arXiv preprint arXiv:1903.12562, 2019.

[14] Li Li. The Calderón problem for the fractional magnetic operator. Inverse Problems, 36(7):075003, 2020.

[15] Li Li. A semilinear inverse problem for the fractional magnetic Laplacian. arXiv preprint arXiv:2005.06714, 2020. 
[16] Gen Nakamura, Ziqi Sun, and Gunther Uhlmann. Global identifiability for an inverse problem for the Schrödinger equation in a magnetic field. Mathematische Annalen, 303(1):377-388, 1995.

[17] Angkana Rüland and Mikko Salo. The fractional Calderón problem: low regularity and stability. Nonlinear Analysis, 193:111529, 2020.

[18] Marco Squassina and Bruno Volzone. Bourgain-Brézis-Mironescu formula for magnetic operators. Comptes Rendus Mathematique, 354(8):825-831, 2016.

[19] Zi Qi Sun. An inverse boundary value problem for Schrödinger operators with vector potentials. Transactions of the American Mathematical Society, 338(2):953-969, 1993. 